\title{
A stable path to ferromagnetic hydrogenated graphene growth
}

\author{
Shayan Hemmatiyan, ${ }^{1}$ Marco Polini, ${ }^{2}$ Artem Abanov, ${ }^{1}$ Allan H. MacDonald,${ }^{3}$ and Jairo Sinova ${ }^{4,1,5}$ \\ ${ }^{1}$ Department of Physics, Texas A\&M University, College Station, TX 77843-4242, USA \\ ${ }^{2}$ NEST, Istituto Nanoscienze - CNR and Scuola Normale Superiore, I-56126 Pisa, Italy \\ ${ }^{3}$ Department of Physics, University of Texas at Austin, Austin, Texas 78712-1081, USA \\ ${ }^{4}$ Institut für Physik, Johannes Gutenberg Universität Mainz, D-55099 Mainz, Germany \\ ${ }^{5}$ Institute of Physics ASCR, Cukrovarnická 10, 16253 Praha 6, Czech Republic
}

(Dated: October 8, 2018)

\begin{abstract}
In this paper, we propose a practical way to stabilize half-hydrogenated graphene (graphone). We show that the dipole moments induced by a hexagonal-boron nitride (h-BN) substrate on graphene stabilize the hydrogen atoms on one sublattice of the graphene layer and suppress the migration of the adsorbed hydrogen atoms. Based upon first principle spin polarized density of states (DOS) calculations, we show that the graphone obtained in different graphene/h-BN heterostructures exhibits a half metallic state. We propose to use this new exotic material for spin valve systems and other spintronics devices.
\end{abstract}

PACS numbers:

\section{INTRODUCTION}

The low electronic density of states near the fermi energy and the atomic thickness of graphene make it a very attractive material for high frequency large-scale integrated electronics 1 . However, due to its semi-metallic nature (zero band-gap at neutrality point), graphene exhibits a small $\mathrm{ON} / \mathrm{OFF}$ switching ratio $(<10$ at room temperature). This problem inhibits the application of graphene for charge based logic devices and integrated circuits.2] 4

One possibility to overcome the small ON/OFF switch ratio is to control charge current via spin. The proposed mechanisms to adjust the current include applying an external magnetic field or a magnetization switching (charge based) via spin polarized current in spin valve systems and tunneling magneto resistance (TMR) devices 5 [ 8 . The magnetic properties of graphene have also been extensively studied in the recent years. ${ }^{[-15}$ It has been shown, that pure graphene exhibits only weak antiferromagnetic (AF) order ${ }^{9}$ at near room temperature. However, studies of structural and other defects show that induced $s p^{3}$-type hybridization, such as monovacancies create a local spin moment and, in certain circumstances, promote a robust long range magnetic order $10 \mid 11$.

A similar effect is obtained by chemical functionalization of the graphene with elements such as hydrogen!10|11]16|17] Maximum magnetic moment $\left(1 \mu_{B}\right.$ per cell) is predicted for the half hydrogenated-graphene (graphone). In such theoretical systems the hydrogen atoms are adsorbed by only one graphene sublattice. Induced $s p^{3}$ hybridization then results in localized magnetic moment in the same way as for defects. However, the overlap between the $p_{z}$ orbitals of the nearest carbons is sufficient to create a long range magnetic order at room temperature 11 . The $p_{z}$ orbitals have a near $3 \mathrm{eV}$ band gap $\sqrt{1819}$ in graphone.

Although graphone could make a breakthrough in spin- tronics, its fabrication is an experimentally challenging task. Fundamentally, there are two obstacles: i) the symmetry between sublattices, and ii) the lack of barrier for the trapped hydrogen atoms to migrate between the sublattices.20

Recently, there has been some progress in fabrication of partially hydrogenated graphene ${ }^{2122}$ under certain experimental conditions (i.e low temperature) 21 . Zhou et al proposed a functionalized heterostructures of fully hydrogenated graphene (graphane) on the top of hexagonalboron nitride (h-BN) 23 . The idea is creating an active nitrogen agent by exposing the system to fluorine. The instability of nitrogen-fluorine bond will increase the electronegativity of nitrogen thus creating an active nitrogen site. By applying pressure on the fluorinated h-BN layer, the system undergoes a structural transition from graphane to semi-hydrogenated graphene by adsorption of all the hydrogen atoms from one sublattice in graphane to h-BN layer $\stackrel{23}{ }$.

Hexagonal boron nitride is the insulating isomorph of graphite (honeycomb lattice with boron and nitrogen on two adjacent sublattices) with a large band gap of $5.97 \mathrm{eV}$. It was shown ${ }^{24}$ that it is a superior substrate for graphene for homogenous and high quality graphene fabrication 2426 . The small lattice mismatching (1.7\%) and atomically planer structure of h-BN (free of dangling bonds and charge traps) preserve properties of graphene such as charge carrier mobility.

In this paper, we present a practical method to solve the two obstacles (symmetry of two sublattices and mobility of hydrogen) using the functionalized graphene hybrid structure with h-BN. Our proposed experimental set up includes two steps: fabrication of graphene on h-BN substrate then exposing the system into the hydrogen plasma. The electrical dipole induced by the substrate in addition to small buckling of carbon bonds will trap hydrogen in one sublattice and will kinematically stabilize the system.

We show that for h-BN the difference in electronega- 
tivity of nitrogen and boron creates a dipole moment for each nitrogen site. This dipole moment breaks the equivalency of two carbon atoms in two different graphene sublattices. The similar screening effect has been reported in multilayer graphene but with different strength 27 . Moreover, the screening effect of h-BN will generate a buckling in the graphene layer. This buckling will change the vertical position of the one sublattice with respect to the other sublattice and will enhance the coverage rate of the hydrogen in one sublattice. The dipole moment is also responsible for the increased migration barrier in the adsorbed hydrogen atoms, effectively pinning the hydrogen atoms to one sublattice.

We also show that the very same dipole moment induced by the h-BN substrate changes the fermi energy of the graphone layer and decreases the band gap of the graphone from near $3 \mathrm{eV}$ in pristine graphone to $1.93 \mathrm{eV}$ in graphone/h-BN heterostructure $\frac{19}{19}$

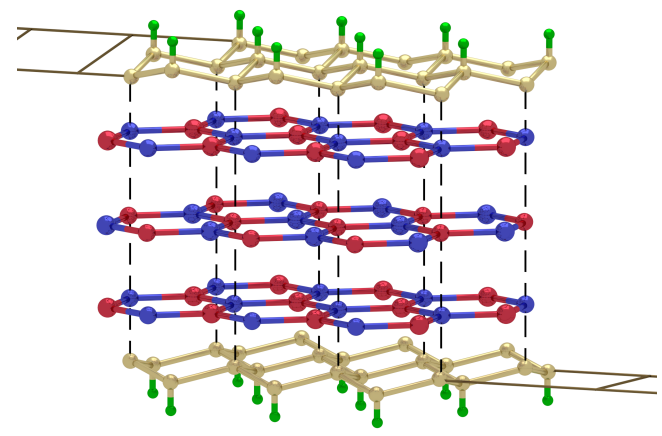

FIG. 1: TMR transistor: Graphone(B)/h-BN/Graphone(B), two ferromagnetic layers of graphone and few (three) layer of h-BN between

We also show that the spin-orbit coupling (SOC) in the graphone increases due to a change in hybridization. In graphene, carbon has small SOC due to its $s p^{2}$ hybridization. The $d$ orbital has the main contribution in creating a $24 \mu \mathrm{eV}$ intrinsic band-gap ${ }^{28}$. However, it has been seen that the hydrogen atom enhances spin orbit interactions in graphene via transition from $s p^{2}$ to $s p^{3}$ type hybridization due to bending of bonds between carbon atoms 29130 . We show this effect will increase in the presence of the h-BN.

Finally, we propose to use the graphone/h-BN heterostructures in TMR devices as shown in Fig. 1. The tunneling current is tuned by changing the magnetization of the one of the electrodes ${ }^{5 / 8}$ We show that multilayer graphone/h-BN heterostructure is a half metal with near $100 \%$ spin polarization. It was speculated before ${ }^{31}$ that such materials are ideal for the TMR devices.

This paper is organized as follows. In II we describe the methods we use. In section [II we show how the screening effect of h-BN depends on stacking. In section IV, we calculate the mobility of hydrogen atoms on both pristine graphene and graphene/h-BN heterostructure. In section
IV, we show that the graphone/h-BN heterostructure is stable. In section V, we perform spin polarized calculations, determine the stable magnetic state, and the magnitude of the SOC. In section VI, we show that not only h-BN heterostructure can be used to trap hydrogen for fabrication of graphone, but could also be utilized as an insulator in TMR devices.

\section{METHODS}

All calculations have been done within the first principle framework of the QUANTUM ESPRESSO package ${ }^{32}$. We utilize both the local density 33 (LDA) and the generalized gradient approximations ${ }^{34}$ (GGA) for graphone on top of h-BN. In addition to GGA, van der Waals (VDW) interactions have been treated through VDW-DF2 $\operatorname{code}^{35}$ within the QUANTUM ESPRESSO package.

It was shown ${ }^{36}$ previously, that LDA is accurate for calculations of the interlayer binding in graphite and multilayer graphene, while GGA better matches the experimental data for intra-band interactions and structure. We note, however, that the discrepancy between the two methods is small and is not essential for the main results of our paper.

LDA calculations are done under ultra-soft, normconserving Perdew-Zunger ${ }^{33}$ (PZ) exchange correlation with the energy cut-off of 60 Ry. For finding optimized structure and activation energy, we used a large supercell, $5 \times 5$ unit cells, to prevent overlapping between distorted areas. Also, a $20 \AA$ vacuum space was used to avoid interactions between two periodic layers.

To find the minimum-energy paths through the migration barrier for the hydrogen atoms, the strong distortion of covalent bonds must be taken into account. For this purpose, we used nudged-elastic-band (NEB) method outlined in Reference 20.

For finding the optimized structure, we used conjugategradient $(\mathrm{CG})$ method with the force and energy convergence parameters $10^{-3}$ Ry/a.u. and $10^{-5}$ Ry, respectively. A mesh of $4 \times 4 \times 1$ k-points in the Mokhorst-Park method ${ }^{37}$ and a cold smearing 38 of $0.01 \mathrm{eV}$ degauss was implemented.

For the calculation of the magnetic properties, we used a spin-polarized LDA (LSDA) with a fully relativistic and norm-conserving Perdew-Burke-Ernzerholf (PBE) method ${ }^{\sqrt{34}}$ which has non-linear core corrections; energy and charge density cut-offs were $50 \mathrm{eV}$ and 500 $\mathrm{eV}$, respectively. We used a non-collinear calculation in the presence of SOC with all spins constrained along the z-direction. Our supercell consists of $2 \times 2$ unit-cells.

To find the SOC, we fit the band structure of graphone/h-BN with SOC to the tight binding model near the Dirac point. A marzari-vanderbilt (m-v) smearing of $0.01 \mathrm{eV}$ has been used for calculating density of states. The force and energy optimization accuracy was $10^{-3} \mathrm{Ry} /$ a.u. and $10^{-5} \mathrm{Ry}$, respectively. 


\section{STACKING GRAPHENE ON H-BN}

In this section, we discuss the different ways of stacking in the graphene/h-BN layers, in which both graphene and h-BN have the hexagonal lattices. The graphene has carbon atoms in both sublattices, while h-BN has boron in one sublattice and nitrogen in the other.

Let us, at the moment, ignore the small (about $1.7 \%$ ) mismatch between the graphene and h-BN lattices. The three most symmetric stacking variations are AA, AB-I, and AB-II: for AA stacking, Fig. 22, each carbon atom of one sublattice of graphene is on top of a boron atom of h-BN, while each carbon atom of the other sublattice of graphene is on top of the nitrogen atom of h-BN; for ABI staking, Fig. 2b, each carbon atom of one sublattice of graphene is on top of a boron atom of h-BN, while each carbon atom of the other sublattice of graphene is on top of the center of a hexagon of h-BN; for AB-I staking, Fig. 2 , each carbon atom of one sublattice of graphene is on top of nitrogen atom of h-BN, while each carbon atom of the other sublattice of graphene is on top of the center of a hexagon of h-BN. First principle calculations $39 \mid 40$ show that AB-II stacking configuration has a much larger energy and thus can be ignored. For the rest of the paper we will use AB to refer to AB-I stacking.

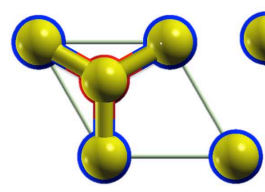

(a)

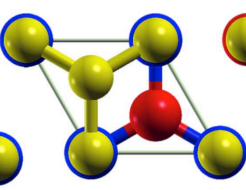

(b)

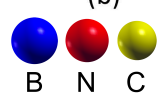

FIG. 2: Different ways of stacking of graphene on top of hBN: a) AA stacking; b) AB-I stacking; b) AB-II stacking. See full description in the text.

Although, the precise stacking control of graphene on $\mathrm{h}-\mathrm{BN}$ is problematic, Wei Yang et $a_{t}^{26}$ showed that epitaxial growth of graphene on h-BN may allow for pure selectional stacking in this heterostructure.

Different stacking types will affect the electrical properties of the substrate, such as the dipole moment, differently. AB stacking provides larger asymmetry between boron and nitrogen environments of h-BN lattice than AA stacking. This difference is taken into account in our calculations.

Consider now a small lattice mismatching $(1.7 \%)$ between graphene and h-BN lattices. This mismatch will create a large moiré pattern as seen in Fig. 3. Given small lattice mismatching and small twisting angle (in the case where we do not have pure AB or AA-stacking), we can divide the supercell into two main stacking domains: AA-stacking at the corners and AB-stacking at $1 / 3$ and $2 / 3$ of the long diagonal $\frac{41}{\text {. The domains fill }}$ most of the area inside the supercells. For each domain, we will have a preference site for hydrogen adsorption on

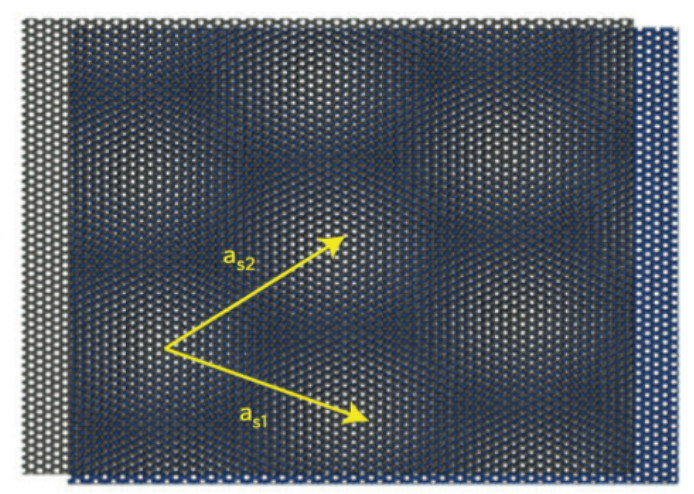

FIG. 3: Creation of moiré pattern in the presence of lattice mismatching between graphene and h-BN sublattice ${ }^{26}$

just one sublattice related to the stacking of the domain.

\section{HYDROGEN ADSORPTION}

In this section, we first show that in the graphene h-BN heterostucture for both stacking $\mathrm{AA}$ and $\mathrm{AB}$, the hydrogen is adsorbed predominantly on one sublattice of the graphene lattice. The half hydrogenated graphene will then normally become perfect graphone. We show that this graphone is stable with respect to hydrogen migration and desorption. We compare the energy differences, migration barriers, migration energies and binding energies of $\mathrm{AA}$ and $\mathrm{AB}$ stacking with that of pristine graphone.

There are three high symmetry adsorption sites per unit cell for pristine graphone and for any stacking types of the heterostucture. The hydrogen atom can be adsorbed on the sublattice A, sublattice B, or in the center of the hexagon $\mathrm{C}$ of graphene, see Fig. 4 .

We use LDA, GGA, and VDW methods to find the energy difference, $\Delta E_{\mathrm{BA}}=E_{\mathrm{A}}-E_{\mathrm{B}}$ and $\Delta E_{\mathrm{BC}}=E_{\mathrm{C}}-$ $E_{\mathrm{B}}$, between different graphones, $\mathrm{A}, \mathrm{B}$, and $\mathrm{C}$, for both stacking types and for the pristine case. The results are

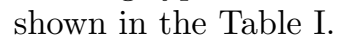

\begin{tabular}{|l|ccc|ccc|}
\hline Config. & \multicolumn{3}{|c|}{$\Delta E_{\mathrm{BA}}$} & \multicolumn{3}{c|}{$\Delta E_{\mathrm{BC}}$} \\
\hline \multirow{3}{*}{ Pristine } & \multicolumn{3}{|c}{ LDA } & 0 & & \multicolumn{3}{c|}{0.02} \\
AB & 0.02 & 0.01 & 0.03 & 0.03 & 0.02 & 0.05 \\
AA & 0.01 & 0.02 & 0.02 & 0.03 & 0.07 & 0.08 \\
\hline
\end{tabular}

TABLE I: Energy differences (in eV) per unit cell, $\Delta E_{\mathrm{BA}}=$ $E_{\mathrm{A}}-E_{\mathrm{B}}$ and $\Delta E_{\mathrm{BC}}=E_{\mathrm{C}}-E_{\mathrm{B}}$ for different types of graphone, A , B, C, pristine graphone and for different kinds of stacking $\mathrm{AB}$ and $\mathrm{AA}$. For three different types of configurations (AA, AB, and pristine graphone) using LDA, GGA, and VDW approximations.

For pristine graphone, where there are no inter-planar VDW interactions, our results reproduce the results of 

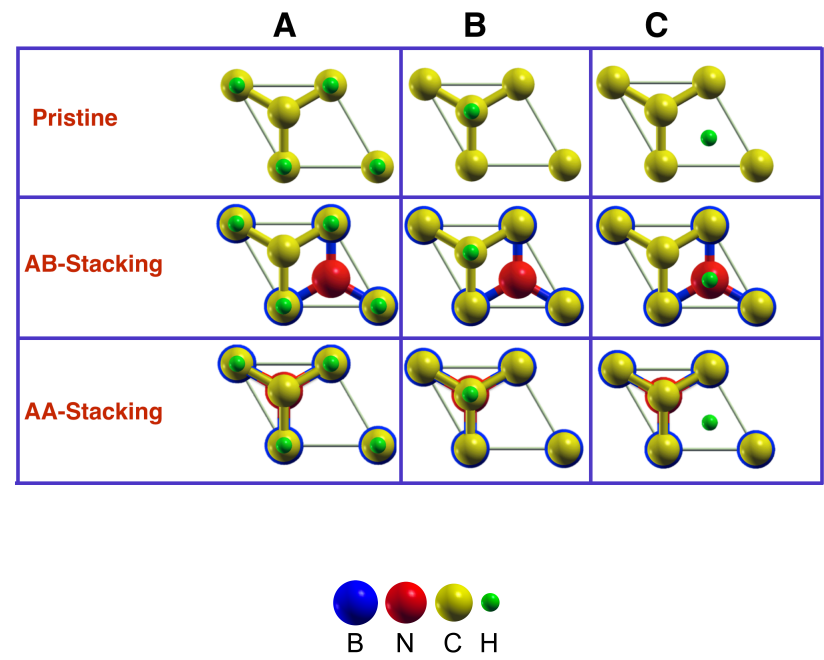

FIG. 4: The first row shows different hydrogenations of graphene, the second row shows different hydrogenations of AB stacking, and the third shows that for AA stacking. Hydrogenation differs by the placement of the hydrogen atom within the unit cell. The first column is graphone(A), the second is graphone $(\mathrm{B})$ and the third is graphone $(\mathrm{C})$. We refer to them in the text as AB-C - this is AB stacked heterostructure which forms graphone $(\mathrm{C})$.

the previous work ${ }^{20}$ which used GGA calculation. It is also no surprise that the graphone(A) and graphone(B) have the same energy, as in the pristine case there is no difference between them. The energy of a pristine graphone $(\mathrm{C})$ is larger then the energy of pristine graphone $(\mathrm{A})$ or $(\mathrm{B})$.

For the graphene h-BN heterostructure the results are different. In both $\mathrm{AA}$ and $\mathrm{AB}$ stacking there is no symmetry between different sublattices. From Table I, we see that the graphone(B) where hydrogen adsorbs to the sublattice B (the sublattice where is not on top of the boron atoms for both $\mathrm{AA}$ and $\mathrm{AB}$ stacking types), has the smallest energy for both ways of stacking. All three methods LDA, GGA, and VDW also show that for both kinds of stacking, graphone $(\mathrm{C})$ has a larger energy than graphone(A).

The conclusion is that the site shown in the column B of Fig. 4 is the preference site for hydrogen adsorption. If the hydrogen atoms adsorb to all sites $\mathrm{B}$ on the hexagonal lattice, the result is pure graphone(B).

We next check the stability of this graphone. First, we check graphone(B) for both ways of stacking against removal of one hydrogen atom or two neighboring hydrogen atoms. The results of LDA, GGA, and VDW calculations for both kinds of stacking as well as for pristine graphone is shown in Table

The conclusion is that graphone(B) is stable against desorption of hydrogen for both $\mathrm{AA}$ and $\mathrm{AB}$ stacking types of the h-BN/graphene heterostructure. We note

\begin{tabular}{|l|lll|lll|}
\hline Config. & \multicolumn{1}{|l|}{ H-Binding energy } & \multicolumn{2}{l|}{ Removal of two H } \\
\hline & \multicolumn{4}{|c|}{ LDA } & GGA VDW & \multicolumn{4}{|c}{ LDA } & GGA VDW \\
Pristine & \multicolumn{4}{|c|}{1.15} & & \multicolumn{4}{c}{-5.31} \\
AB & 1.10 & 1.01 & 1.12 & 0.32 & 0.36 & 0.38 \\
AA & 1.09 & 1.01 & 1.10 & 0.31 & 0.33 & 0.34 \\
\hline
\end{tabular}

TABLE II: Binding energy for one and two neighboring hydrogen atoms for graphone(B) for pristine, AA, and AB stacking variations. The energies are given in $\mathrm{eV}$ per unit-cell.

that the pristine graphone is not stable against desorption of two neighboring hydrogen atoms.

Finally, we check the stability of graphone(B) against migration of the hydrogen atom to a nearest site on another sublattice. In order to do that, we calculate both the migration energy and migration barrier. We denote these graphone types as graphone(A), graphone(B) and graphone $(\mathrm{C})$.

For finding the migration energy, one hydrogen atom is moved per supercell $(5 \times 5$ unit-cell $)$ to the nearby sublattice and then the energy difference per unit-cell for these two configurations is found. The migration barrier has been derived by the minimum-energy path calculation under the NEB method ${ }^{42}$ NEB results for four different supercells, $2 \times 2,3 \times 3,4 \times 4$ and $5 \times 5$ indicate a fairly small (less than $5 \%$ ) correlation effect of two distorted adjacent supercells. For pristine graphone, only the GGA calculation was performed and results are in agreement with the previous work 20

\begin{tabular}{|l|c|ccc|}
\hline Config. & Migration barrier & \multicolumn{2}{|c|}{ Migration energy } \\
\hline & LDA (NEB) & \multicolumn{2}{|c}{ LDA GGA VDW } \\
Pristine & 0.06 & \multicolumn{2}{|c}{-1.44} & \\
AB & 0.18 & -0.87 & -1.04 & -0.64 \\
AA & 0.12 & -0.93 & -1.12 & -0.82 \\
\hline
\end{tabular}

TABLE III: Migration barrier and migration energy for three different types of configurations.

It was previously shown ${ }^{20}$ that the small migration barrier makes the pristine graphone unstable against hydrogen migration. Indeed we see in Table III that this barrier is just $0.06 \mathrm{eV}$. The table also shows a substantial increase in the migration barrier for both AA and $\mathrm{AB}$ stacking. This increase is due to the screening effect of h-BN.

Such barriers will decrease the mobility of hydrogen atoms on top of the graphene layer in the presence of h-BN. If we start with half hydrogenated graphene, from the Arrhenius equation, $e^{\left(-\frac{\Delta E}{k_{B} T}\right)}$, at room temperature, the transition probability will be less than 0.001 and from $\tau \sim \frac{\hbar}{k_{B} T} e^{\left(\frac{\Delta E}{k_{B} T}\right)}$ the naive estimate for transition time will be $10^{-10} \mathrm{sec}$. The transition time increases rapidly by decreasing temperature whereas at $T=60 \mathrm{~K}$, the transition time reaches to the order of $1 \mathrm{sec}$.

The migration energy for both types of stacking is still negative, although substantially less than for pristine graphone. Although this still makes the boat gra- 
phone (see Fig. 5 the most stable configuration thermodynamically, if one exposes the graphene $/ \mathrm{hBN}$ to the hydrogen plasma, graphone(B) will be the most stable case kinematically. This can be understood from the fact that carbon atoms belonging to sublattice B come first in contact with hydrogen atoms and they then encounter a large migration barrier due to the presence of the substrate. Moreover, it has been shown recently $19 \sqrt{193}$ that half-hydrogenated graphene only in one sublattice can be fabricated within the selective desorption of hydrogen atoms from one side of the chair graphone 44 . Such produced graphone then will be stabilized by the high migration barrier for the h-BN/graphene heterostructure.
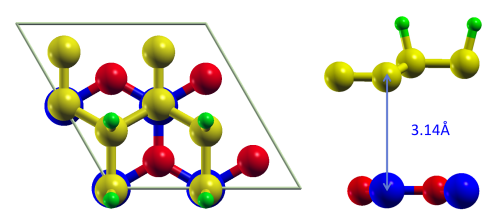

FIG. 5: Optimized structure for boat graphone/h-BN with $2 \times 2$ supercell.

\section{MAGNETIC PROPERTIES OF GRAPHONE/H-BN}

One of the main reasons why pristine graphone(A) or graphone(B) are interesting and promising is the magnetic properties of these materials. It has been shown by spin-polarized first principle calculation in LSDA scheme that graphone (A or B) has about $1 \mu_{B}$ per unit-cell magnetization 16 . Unfortunately, pristine graphone is not stable enough to be of any practical use.

In the previous section, we showed that h-BN substrate considerably increases the graphone(B) stability, making it feasible for further studies. It is, however, important to show that such stabilized graphone still has the magnetic properties expected from the pristine graphone. In this section, we present the results of our LSDA with GGA calculations for graphone(B) on h-BN substrate.

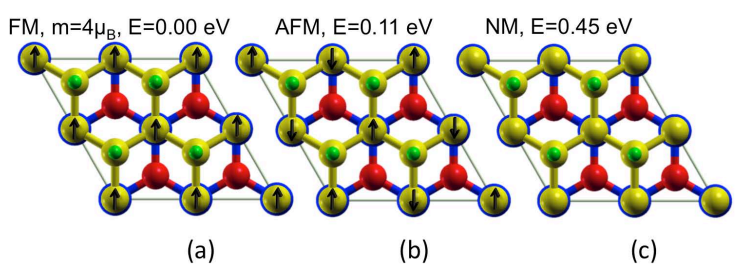

FIG. 6: Different magnetization states of graphone(B)/h-BN with their relative energy: a) ferromagnetic with $1 \mu_{B}$ per unit-cell magnetization which is the most stable configuration, b) AFM state with zero magnetization, and c) normal state.

Our calculations were done with fully relativistic PBEGGA pseudo potential with non-linear core corrections and cold smearing with $0.001 \mathrm{eV}$ degauss. Our supercell consists of $2 \times 2$ unit-cells with $20 \stackrel{\circ}{A}$ vacuum layer and $16 \times 16 \times 1$ Monkhorst-Pack k-points mesh. The result shows about $1 \mu_{B}$ magnetization and a $0.42 \mathrm{eV}$ (per unitcell) energy difference between the ferromagnetic state (FM) and the normal state, see Fig. 6.

The band structure of the optimized graphone(B)/h$\mathrm{BN}$ is shown in Fig. 7. Group symmetry near K-point and $\Gamma$-point is $C_{3}$ and $C_{3 \nu}$ respectively, whereas the $\pi^{*}$ band is located in the vicinity of the fermi energy $\frac{30}{\mathrm{Up}}$ to the first order in momentum near the $\mathrm{K}$-point and $\Gamma$ point, these bands are described by the following SOC Hamiltonians:

$$
\begin{gathered}
H_{\text {SOC }}^{\tau K}=\lambda^{\mathrm{BR}}\left(k_{x} s_{y}-k_{y} s_{x}\right)+\tau \lambda^{\mathrm{I}} s_{z} \\
H_{\text {SOC }}^{\Gamma}=\lambda^{\mathrm{BR}}\left(k_{x} s_{y}-k_{y} s_{x}\right)
\end{gathered}
$$

(b)

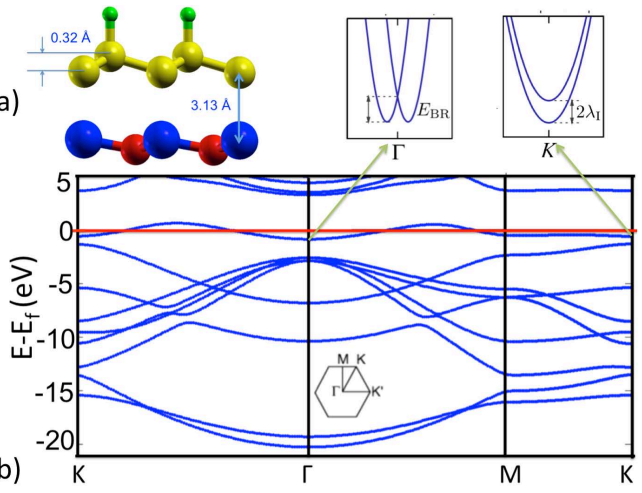

FIG. 7: a) Optimized structure for graphone(B)/h-BN b) Band structure of graphone/h-BN (fully relativistic GGA) with considering SOC.

The two terms in Eq. (2), correspond to induced Bychkov-Rashba-like SOC with $\lambda^{\mathrm{BR}}=0.1 \mathrm{meV}$ and intrinsic SOC with $\lambda^{\mathrm{I}}=1.2 \mathrm{meV}$, respectively. $\tau$ is +1 $(-1)$ for the $\mathrm{K}\left(\mathrm{K}^{\prime}\right)$ point. $\lambda^{\mathrm{I}}$ is half of the band splitting of $\pi^{*}$ band in the vicinity of fermi energy at the K point $(2.4 \mathrm{meV})$ and $\lambda^{\mathrm{BR}}$ was obtained at the $\Gamma$ point.

The increase of $\lambda^{\mathrm{I}}$ in comparison to $\lambda^{\mathrm{I}}=12 \mu \mathrm{eV}$ for graphene ${ }^{28}$ is due to the bending of the carbon bonds, see Fig. 7. This bending changes the $s p^{2}$, plane graphene, hybridization to $s p^{3}$, diamond like, hybridization. This value of $\lambda^{\mathrm{I}}$ is comparable to fully hydrogenated graphene in both sides (chair graphane ${ }^{29}$ ), which reported to have $8.7 \mathrm{meV}$ band splitting.

\section{TMR DEVICE: GRAPHONE/H-BN/GRAPHONE}

Tunneling magneto resistance occurs in magnetic tunnel junctions that consist of two ferromagnetic metallic 
layers with a good insulator in between. The tunneling current is then controlled by switching magnetization in one of the layers. It is important for these devices that the ferromagnetic layers have highly spin polarized electronic states near the fermi energy. In this section, we show that graphone/h-BN multilayer heterostructures possess nearly $100 \%$ polarized states at the fermi energy and thus are perfect half metals. We also show that the magnetic properties of the graphone can be controlled and enhanced by changing the number of layers of h-BN. h-BN has a large band gap $(6 \mathrm{eV})$ and can be a good insulator for fabrication of TMR devices. These properties of the multilayer graphene h-BN heterostructures can open new horizons in spintronics.

We perform the same LDA, GGA, and VDW structure calculation for two layers of graphone(B) with 1, 2, and 3 layers of AA-stacked h-BN sandwiched in between. The results are shown in Table IV.

\begin{tabular}{|c|cc|cc|cc|}
\hline Structure & LDA & & GGA & & \multicolumn{2}{l|}{ VDW } \\
& $d_{C B}$ & $d_{C C}^{\perp}$ & $d_{C B}$ & $d_{C C}^{\perp}$ & $d_{C B}$ & $d_{C C}^{\perp}$ \\
\hline graphone & 3.12 & 0.38 & 3.13 & 0.30 & 3.13 & 0.32 \\
g/1bn/g & 2.60 & 0.28 & 2.72 & 0.29 & 3.19 & 0.30 \\
g/2bn/g & 1.95 & 0.38 & 2.10 & 0.38 & 3.08 & 0.30 \\
g/3bn/g & 2.48 & 0.28 & 2.47 & 0.30 & 3.01 & 0.31 \\
\hline
\end{tabular}

TABLE IV: Optimized structure (in $\AA$ ): $d_{C B}$ is the distance between carbon on top of the boron atom and $d_{c c}^{\perp}$ is the vertical distance between two carbon atoms which belong to the same layer. It shows the magnitude of the bond bending.

We calculated the spin polarized density of states for the three multilayer structures using LSDA scheme with $0.01 \mathrm{eV}$ degauss and cold smearing. The structural parameters for this calculations was taken from the VDW results. The results are shown in Fig. 8.

All three heterostructures show half metallic behavior near the fermi-energy. The spin up gap varies between 1 and $2 \mathrm{eV}$. According to Julliere mode ${ }^{31}$, such half metallic materials with $100 \%$ spin polarization are ideal for fabrication of TMR devises.

\section{CONCLUSION}

We proposed a feasible way to fabricate graphone in experiment by exposing the graphene/h-BN bilayer to the hydrogen plasma. The dipole moments induced by h-BN orchestrates the hydrogen pattern on the graphene layer. From first principle calculations, we have shown the presence of the preference site for hydrogen adsorption and an increment in migration barrier due to the screening effect of h-BN. The results show induced dipole moments by h-BN will trap hydrogen atoms to only one sublattice and will also kinematically stabilize the graphone layer.

The calculated band structures for the optimized graphone(B) on h-BN shows that the screening effect of h-BN not only reduces the band gap (from near $3 \mathrm{eV}$ in pristine graphone to $1.93 \mathrm{eV}$ in the graphone/h-BN
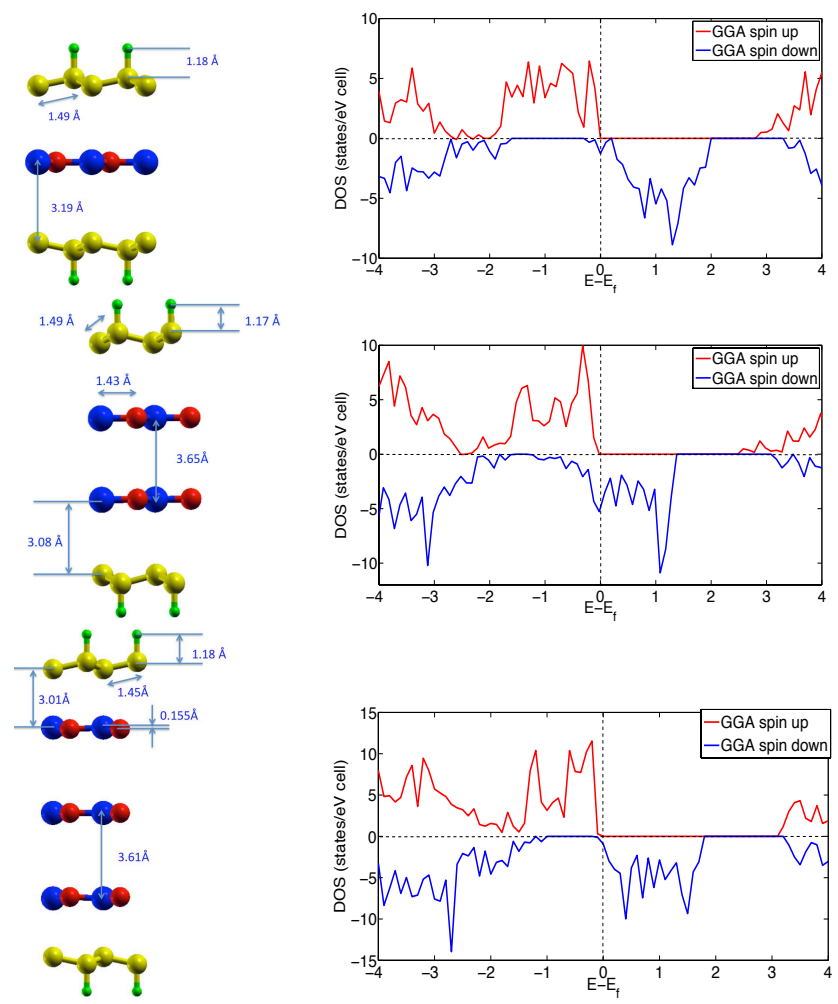

FIG. 8: Optimized structure and spin-polarized DOS (all the energies are in $\mathrm{eV}$ ) for double-layered graphone(B) and a) single , b) double and c) triple-layered AA-stacked h-BN in between.

heterostructure ${ }^{19}$ ), but will also effectively change the fermi energy in the graphone layer.

Finally, we have shown that multilayer heterostructures (several layers of h-BN are sandwiched in between two layers of graphone) are half metal with near $100 \%$ spin polarization. We propose to use such heterostructures in TMR devices.

We note, that it is also possible to use different elements such as fluorine as an adsorbent on graphene/h-BN heterostructures. These new materials will have different binding energies, migration barriers, and electronic properties. Doping h-BN will change the fermi energy of the graphone layer in addition to affecting hydrogen pattern on the graphene lattice. Therefore, it may provide the mechanism for control of the hydrogen pattern and electronic properties of the heterostructures.

\section{Acknowledgments}

The authors gratefully acknowledge Cristian Cernov. This work was supported by SWAN, DMR-1105512, onrn000141110780, and Alexander Von Humboldt Foundation. Ar. Abanov was supported by Welch Foundation (A-1678). 
1 Andre K Geim and Konstantin S Novoselov. The rise of graphene. Nature materials, 6(3):183-191, 2007.

2 L. Britnell, R. V. Gorbachev, R. Jalil, B. D. Belle, F. Schedin, A. Mishchenko, T. Georgiou, M. I. Katsnelson, L. Eaves, S. V. Morozov, N. M. R. Peres, J. Leist, A. K. Geim, K. S. Novoselov, and L. A. Ponomarenko. Field-effect tunneling transistor based on vertical graphene heterostructures. Science, 335(6071):947-950, 2012.

3 Shayan Hemmatiyan, Xingyuan Pan, Marco Polini, Allan MacDonald, and Jairo Sinova. Vertical transport properties of graphene/h-bn hetrostructures. In APS March Meeting Abstracts, volume 1, page 7003, 2013.

4 Xingyuan Pan, Shayan Hematiyan, Jairo Sinova, Marco Polini, and Allan MacDonald. Vertical transport through twisted graphene/h-bn heterostructure. Bulletin of the American Physical Society, 58, 2013.

5 S. Il Kiselev, J. C. Sankey, I. N. Krivorotov, N. C. Emley, R. J. Schoelkopf, R. A. Buhrman, and D. C. Ralph. Microwave oscillations of a nanomagnet driven by a spinpolarized current. Nature, 425(6956):380-383, 2003.

${ }^{6}$ G. Bertotti, C. Serpico, I. D. Mayergoyz, A. Magni, M. d'Aquino, and R. Bonin. Magnetization switching and microwave oscillations in nanomagnets driven by spinpolarized currents. Phys. Rev. Lett, 94(12):127206, 2005.

7 Christian Heiliger and M. D. Stiles. Ab Initio studies of the spin-transfer torque in magnetic tunnel junctions. Phys. Rev. Lett., 100:186805, May 2008.

8 J. S. Moodera, Lisa R. Kinder, Terrilyn M. Wong, and R. Meservey. Large magnetoresistance at room temperature in ferromagnetic thin film tunnel junctions. Phys. Rev. Lett., 74:3273-3276, Apr 1995.

9 S. Sorella and E. Tosatti. Semi-metal-insulator transition of the hubbard model in the honeycomb lattice. EPL (Europhysics Letters), 19(8):699, 2007.

10 Oleg V. Yazyev and Lothar Helm. Defect-induced magnetism in graphene. Phys. Rev. B, 75:125408, Mar 2007.

11 Oleg V Yazyev. Emergence of magnetism in graphene materials and nanostructures. Reports on Progress in Physics, 73(5):056501, 2010.

12 J. J. Palacios, J. Fernández-Rossier, and L. Brey. Vacancyinduced magnetism in graphene and graphene ribbons. Phys. Rev. B, 77:195428, May 2008.

13 M. Topsakal, E. Aktürk, H. Sevinçli, and S. Ciraci. Firstprinciples approach to monitoring the band gap and magnetic state of a graphene nanoribbon via its vacancies. Phys. Rev. B, 78:235435, Dec 2008.

14 Yang Lei, Stephen A. Shevlin, Wenguang Zhu, and Zheng Xiao Guo. Hydrogen-induced magnetization and tunable hydrogen storage in graphitic structures. Phys. Rev. B, 77:134114, Apr 2008.

15 X. Y. Cui, R. K. Zheng, Z. W. Liu, L. Li, B. Delley, C. Stampfl, and S. P. Ringer. Magic numbers of nanoholes in graphene: Tunable magnetism and semiconductivity. Phys. Rev. B, 84:125410, Sep 2011.

16 J. Zhou, Q. Wang, Q. Sun, X. S. Chen, Y. Kawazoe, and P. Jena. Ferromagnetism in semihydrogenated graphene sheet. Nano letters, 9(11):3867-3870, 2009.

17 Mohammed Moaied, J. V. Alvarez, and J. J. Palacios. Hydrogenation-induced ferromagnetism on graphite surfaces. arXiv preprint arXiv:1405.3168, 2014.

18 G. Fiori, S. Lebègue, A. Betti, P. Michetti, M. Klinten- berg, O. Eriksson, and G. Iannaccone. Simulation of hydrogenated graphene field-effect transistors through a multiscale approach. Phys. Rev. B, 82:153404, Oct 2010.

19 Neerav Kharche and Saroj K Nayak. Quasiparticle band gap engineering of graphene and graphone on hexagonal boron nitride substrate. Nano letters, 11(12):5274-5278, 2011.

20 DW Boukhvalov. Stable antiferromagnetic graphone. Physica E: Low-dimensional Systems and Nanostructures, 43(1):199-201, 2010.

21 Qing Peng, AK Dearden, J Crean, L Han, S Liu, X Wen, and S De. New materials graphyne, graphdiyne, graphone, and graphane: review of properties, synthesis, and application in nanotechnology. Nanotechnology, Science and Applications, 7:1-29, 2014.

22 A. J. M. Giesbers, K. Uhlírová, M. Konečnỳ, E. C. Peters, M. Burghard, J. Aarts, and C. F. J. Flipse. Interface-induced room-temperature ferromagnetism in hydrogenated epitaxial graphene. Physical review letters, 111(16):166101, 2013.

23 Jian Zhou and Qiang Sun. How to fabricate a semihydrogenated graphene sheet? a promising strategy explored. Applied Physics Letters, 101(7):073114, 2012.

${ }^{24}$ CR Dean, AF Young, I Meric, C Lee, L Wang, S Sorgenfrei, K Watanabe, T Taniguchi, P Kim, KL Shepard, et al. Boron nitride substrates for high-quality graphene electronics. Nature nanotechnology, 5(10):722-726, 2010.

25 Soo Min Kim, Allen Hsu, PT Araujo, Yi-Hsien Lee, Tomáås Palacios, Mildred Dresselhaus, Juan-Carlos Idrobo, Ki Kang Kim, and Jing Kong. Synthesis of patched or stacked graphene and hbn flakes: A route to hybrid structure discovery. Nano letters, 13(3):933-941, 2013.

26 Wei Yang, Guorui Chen, Zhiwen Shi, Cheng-Cheng Liu, Lianchang Zhang, Guibai Xie, Meng Cheng, Duoming Wang, Rong Yang, Dongxia Shi, et al. Epitaxial growth of single-domain graphene on hexagonal boron nitride. $\mathrm{Na}$ ture materials, 12(9):792-797, 2013.

27 Mohammed Moaied, J. A. Moreno, M. J. Caturla, and J. J. Palacios. A theoretical study of the dynamics of atomic hydrogen adsorbed on graphene multilayers. arXiv preprint arXiv:1405.3165, 2014.

28 Sergej Konschuh, Martin Gmitra, and Jaroslav Fabian. Tight-binding theory of the spin-orbit coupling in graphene. Phys. Rev. B, 82:245412, Dec 2010.

29 Jian Zhou, Qifeng Liang, and Jinming Dong. Enhanced spin-orbit coupling in hydrogenated and fluorinated graphene. Carbon, 48(5):1405-1409, 2010.

30 Martin Gmitra, Denis Kochan, and Jaroslav Fabian. Spinorbit coupling in hydrogenated graphene. Phys. Rev. Lett., 110:246602, Jun 2013.

31 Michel Julliere. Tunneling between ferromagnetic films. Physics Letters A, 54(3):225-226, 1975.

32 Paolo Giannozzi, Stefano Baroni, Nicola Bonini, Matteo Calandra, Roberto Car, Carlo Cavazzoni, Davide Ceresoli, Guido L Chiarotti, Matteo Cococcioni, Ismaila Dabo, Andrea Dal Corso, Stefano de Gironcoli, et al. Quantum espresso: a modular and open-source software project for quantum simulations of materials. Journal of Physics: Condensed Matter, (39):395502 (19pp).

33 J. P. Perdew and Alex Zunger. Self-interaction correction to density-functional approximations for many-electron 
systems. Phys. Rev. B, 23:5048-5079, May 1981.

34 John P. Perdew, Kieron Burke, and Matthias Ernzerhof. Generalized gradient approximation made simple. Phys. Rev. Lett., 77:3865-3868, Oct 1996.

35 D. C. Langreth, Bengt I Lundqvist, Svetla D ChakarovaKäck, V. R. Cooper, Max Dion, Per Hyldgaard, A. Kelkkanen, Jesper Kleis, Lingzhu Kong, Shen Li, et al. A density functional for sparse matter. Journal of Physics: Condensed Matter, 21(8):084203, 2009.

36 D. W. Boukhvalov, M. I. Katsnelson, and A. I. Lichtenstein. Hydrogen on graphene: Electronic structure, total energy, structural distortions and magnetism from firstprinciples calculations. Phys. Rev. B, 77:035427, Jan 2008.

37 Hendrik J Monkhorst and James D Pack. Special points for brillouin-zone integrations. Physical Review B, 13(12):5188-5192, 1976.

38 Nicola Marzari, David Vanderbilt, Alessandro De Vita, and M. C. Payne. Thermal contraction and disordering of the al(110) surface. Phys. Rev. Lett., 82:3296-3299, Apr 1999.
39 Gianluca Giovannetti, Petr A. Khomyakov, Geert Brocks, Paul J. Kelly, and Jeroen van den Brink. Substrateinduced band gap in graphene on hexagonal boron nitride: Ab initio density functional calculations. Phys. Rev. B, 76:073103, Aug 2007.

40 J Sławińska, I Zasada, and Z Klusek. Energy gap tuning in graphene on hexagonal boron nitride bilayer system. Physical Review B, 81(15):155433, 2010.

${ }^{41}$ G Trambly de Laissardière, Didier Mayou, and Laurence Magaud. Localization of dirac electrons in rotated graphene bilayers. Nano letters, 10(3):804-808, 2010.

42 T. O. Wehling, M. I. Katsnelson, and A. I. Lichtenstein. Impurities on graphene: Midgap states and migration barriers. Phys. Rev. B, 80:085428, Aug 2009.

43 Martin Pumera and Colin Hong An Wong. Graphane and hydrogenated graphene. Chemical Society Reviews, 2013.

44 Jorge O. Sofo, Ajay S. Chaudhari, and Greg D. Barber. Graphane: A two-dimensional hydrocarbon. Phys. Rev. B, 75:153401, Apr 2007. 